\title{
395.
}

\section{INVESTIGATIONS IN CONNEXION WITH CASEY'S EQUATION.}

[From the Quarterly Journal of Pure and Applied Mathematics, vol. viII. (1867), pp. $334-341$.]

IN a paper read April 9, 1866, and recently published in the Proceedings of the Royal Irish Academy, Mr Casey has given in a very elegant form the equation of a pair of circles touching each of three given circles, viz. if $U=0, V=0, W=0$ be the equations of the three given circles respectively, and if considering the common tangents of $(V=0, W=0)$, of $(W=0, U=0)$, and of $(U=0, V=0)$ respectively, these common tangents being such that the centres of similitude through which they respectively pass lie in a line (viz. the tangents are all three direct, or one is direct and the other two are inverse), then if $f, g, h$ are the lengths of the tangents in question, the equation

$$
\sqrt{ }(f U)+\sqrt{ }(g V)+\sqrt{ }(h W)=0,
$$

belongs to a pair of circles, each of them touching the three given circles. (There are, it is clear, four combinations of tangents, and the theorem gives therefore the equations of four pairs of circles, that is of the eight circles which touch the three given circles.)

Generally, if $U=0, V=0, W=0$ are the equations of any three curves of the same order $n$, and if $f, g, h$ are arbitrary coefficients, then the equation

$$
\sqrt{ }(f U)+\sqrt{ }(g V)+\sqrt{ }(h W)=0,
$$

is that of a curve of the order $2 n$, touching each of the curves $U=0, V=0, W=0$, $n^{2}$ times, viz. it touches

$$
\begin{aligned}
& U=0 \text {, at its } n^{2} \text { intersections with } g V-h W=0 \text {, } \\
& V=0 \quad \text { " } \quad h W-f U=0 \text {, } \\
& W=0 \quad \text { \# } \quad, \quad f U-g V=0 .
\end{aligned}
$$

C, VI. 
If however the curves $U=0, V=0, W=0$ have a common intersection, then the curve in question has a node at this point, and besides touches each of the three curves in $n^{2}-1$ points; and similarly, if the curves $U=0, V=0, W=0$ have $k$ common intersections, then the curve in question has a node at each of these points, and besides touches each of the three curves in $n^{2}-k$ points.

In particular, if $U=0, V=0, W=0$ are conics having two common intersections, then the curve is a quartic having a node at each of the common intersections, and besides touching each of the given conics in two points; whence, if the coefficients $f, g, h$ (that is, their ratios) are so determined that the quartic may have two more nodes, then the quartic, having in all four nodes, will break up into a pair of conics, each passing through the common intersections, and the pair touching each of the given conics in two points; that is, the component conics will each of them touch each of the given conics once. Taking the circular points at infinity for the common intersections, the conics will be circles, and we thus see that Casey's theorem is in effect a determination of the coefficients $f, g, h$, in such wise that the curve

$$
\sqrt{ }(f U)+\sqrt{ }(g V)+\sqrt{ }(h W)=0,
$$

(which when $U=0, V=0, W=0$ are circles, is by what precedes a bicircular quartic) shall have two more nodes, and so break up into a pair of circles.

The question arises, given $U=0, V=0, W=0$, curves of the same order $n$, it is required to determine the ratios $f: g: h$ in such wise that the curve

$$
\sqrt{ }(f U)+\sqrt{ }(g V)+\sqrt{ }(h W)=0,
$$

may have two nodes; or we may simply inquire as to the number of the sets of values of $(f: g: h)$, which give a binodal curve, $\sqrt{ }(f U)+\sqrt{ }(g V)+\sqrt{ }(h W)=0$.

I had heard of Mr Casey's theorem from Dr Salmon, and communicated it together with the foregoing considerations to Prof. Cremona, who, in a letter dated Bologna, March 3, 1866, sent me an elegant solution of the question as to the number of the binodal curves. This solution is in effect as follows:

Lemma. Given the curves $U=0, V=0, W=0$ of the same order $n$; consider the point $(f, g, h)$, and corresponding thereto the curve $f U+g V+h W=0$. As long as the point $(f, g, h)$ is arbitrary, the curve $f U+g V+h W=0$, will not have any node, and in order that this curve may have a node, it is necessary that the point $(f, g, h)$ shall lie on a certain curve $\Sigma$; this being so, the node will lie on a curve $J$, the Jacobian of the curves $U, V, W$; and the curves $J$ and $\Sigma$ will correspond to each other, point to point; viz. taking for $(f, g, h)$ any point whatever on the curve $\Sigma$, the curve $f U+g V+h W=0$ will be a curve having a node at some one point on the curve $J$; and conversely, in order that the curve $f U+g V+h W=0$ may be a curve having a node at a given point on the curve $J$, it is necessary that the point $(f, g, h)$ shall be at some one point of the curve $\Sigma$. The curve $\Sigma$ has however nodes and cusps; each node of $\Sigma$ corresponds to two points of $J$, viz. the point $(f, g, h)$ being at a node of $\Sigma$, the curve $f U+g V+h W=0$, is a binodal curve having a node at 
each of the corresponding points on $J$; and each cusp of $\Sigma$ corresponds to two coincident points of $J$, viz. the point $(f, g, h)$ being at a cusp of $\Sigma$, the curve $f U+g V+h W=0$ is a cuspidal curve having a cusp at the corresponding point of $J$. The number of the binodal curves $f U+g V+h W=0$ is thus equal to the number of the nodes of $\Sigma$, and the number of the cuspidal curves $f U+g V+h W=0$ is equal to the number of the cusps of $\Sigma$. The curve $\Sigma$ is easily shown to be a curve of the order $3(n-1)^{2}$ and class $3 n(n-1)$; and quà curve which corresponds point to point with $J$, it is a curve having the same deficiency as $J$, that is a deficiency $=\frac{1}{2}(3 n-4)(3 n-5)$; we have thence the Plïckerian numbers of the curve $\Sigma$, viz.

$$
\begin{array}{ll}
\text { Order is } & =3(n-1)^{2}, \\
\text { Class } & =3 n(n-1), \\
\text { Cusps } & =12(n-1)(n-2), \\
\text { Nodes } & =\frac{3}{2}(n-1)(n-2)\left(3 n^{2}-3 n-11\right), \\
\text { Inflexions } & =3(n-1)(4 n-5), \\
\text { Double tangents } & =\frac{3}{2}(n-1)(n-2)\left(3 n^{2}+3 n-8\right) .
\end{array}
$$

Remarks. The consideration of the foregoing curve $\Sigma$ is, I believe, first due to Prof. Cremona, it is a curve related to the three distinct curves $U=0, V=0, W=0$, in the same way precisely as Steiner's curve $P_{0}$ is related to the three curves $d_{x} U=0, d_{y} U=0, d_{z} U=0$. (Steiner, "Allgemeine Eigenschaften der algebraischen Curven," Crelle, t. XLVII. (1854), pp. 1-6; see also Clebsch, "Ueber einige von Steiner behandelte Curven," Crelle, t. LxIv. (1865), pp. 288-293), and the Plückerian numbers of $P_{0}$ (writing therein $n+1$ for $n$ ) are identical with those of $\Sigma$. The foregoing expressions $\frac{3}{2}(n-1)(n-2)\left(3 n^{2}-3 n-11\right)$ and $12(n-1)(n-2)$ for the numbers of the binodal and cuspidal curves $f U+g V+h W=0$, are given in my memoir "On the Theory of Involution," Cambridge Philosophical Transactions, t. XI. (1866), pp. 21-38, see p. 32, [348]; but the employment of the curve $\Sigma$ very much simplifies the investigation.

Passing now to the proposed question, we have as before the curves $U=0, V=0, W=0$, of the same order $n$; and we may consider the point $(f, g, h)$, and corresponding thereto the curve $\sqrt{ }(f U)+\sqrt{ }(g V)+\sqrt{ }(h W)=0$, say for shortness the curve $\Omega$, which is a curve of the order $2 n$, having $n^{2}$ contacts with each of the given curves $U, V, W$. As long as the point $(f, g, h)$ is arbitrary, the curve $\Omega$ has not any node; and in order that this curve may have a node, it is necessary that the point $(f, g, h)$ shall lie on a certain curve $\Delta$; this being so, the node will lie on the foregoing curve $J$, the Jacobian of the given curves $U, V, W$; and the curves $J$ and $\Delta$ will correspond to each other, point to point, viz. taking for $(f, g, h)$ any point whatever on the curve $\Delta$, the curve $\Omega$ will have a node at some one point of $J$; and conversely, in order that the curve $\Omega$ may be a curve having a node at a given point of $J$, it is necessary that the point $(f, g, h)$ shall be at some one point of the curve $\Delta$. The curve $\Delta$ has however nodes and cusps; each node of $\Delta$ corresponds to two points of $J$, viz. for $(f, g, h)$ at a node of $\Delta$, the curve $\Omega$ is a binodal curve having a node at each of the corresponding points of $J$; each cusp of $\Delta$ corresponds to two coincident points of $J$, viz. for $(f, g, h)$ at a cusp of $\Delta$, the curve $\Omega$ is a cuspidal curve having a cusp at the corresponding $9-2$ 
point of $J$. The number of the binodal curves $\Omega$ is consequently equal to that of the nodes of $\Delta$, and the number of the cuspidal curves $\Omega$ is equal to that of the cusps of $\Delta$; we have consequently to find the Plückerian numbers of the curve $\Delta$; and this Prof. Cremona accomplishes by bringing it into connexion with the foregoing curve $\Sigma$, and making the determination depend upon that of the number of the conics which satisfy certain conditions of contact in regard to the curve $\Sigma$.

Consider, as corresponding to any given point $(f, g, h)$ whatever, the conic $\frac{f}{x}+\frac{g}{y}+\frac{h}{z}=0$ which passes through three fixed points, the angles of the triangle $x=0, y=0, z=0$. For points $(f, g, h)$ which lie in an arbitrary line $A f+B g+C h=0$, the corresponding conics pass through the fourth fixed point $x: y: z=A: B: C$. Assume for the moment that to the points $(f, g, h)$ which lie on the foregoing curve $\Delta$, correspond conics which touch the foregoing curve $\Sigma$. Then $1^{\circ}$. to the points of intersection of the curve $\Delta$ with an arbitrary line, correspond the conics which pass through four arbitrary points and touch the curve $\Sigma$; or the order of the curve $\Delta$ is equal to the number of the conics which can be drawn through four arbitrary points to touch the curve $\Sigma$; viz. if $m$ be the order, $n$ the class of $\Sigma$, the number of these conics is $=2 m+n$, or substituting for $m, n$ the values $3(n-1)^{2}$ and $3 n(n-1)$ respectively, the number of these conics, that is the order of $\Delta$, is $=3(n-1)(3 n-2)$. $2^{\circ}$. To the nodes of $\Delta$ correspond the conics which pass through three arbitrary points and have two contacts with $\Sigma$, viz. if $m$ be the order, $n$ the class, and $\kappa$ the number of cusps of $\Sigma$, then the number of these conics is $=\frac{1}{2}(2 m+n)^{2}-2 m-5 n-\frac{3}{2} \kappa$, or substituting for $m, n$ their values as above, and for $\kappa$ its value $=12(n-1)(n-2)$, the number of these conics, that is, the number of the nodes of $\Delta$, is found to be

$$
=\frac{3}{2}(n-1)\left(27 n^{3}-63 n^{2}+22 n+16\right) \text {. }
$$

$3^{\circ}$. To the cusps of $\Delta$ correspond the conics which pass through three arbitrary points, and have with $\Sigma$ a contact of the second order; the number of these $(m, n, \kappa$ as above) is $=3 n+\kappa$, or substituting for $n$ and $\bar{\kappa}$ their values as above, the number of these conics, that is the number of the cusps of $\Delta$, is $=3(n-1)(7 n-8)$. We have thence all the Plückerian numbers of the curve $\Delta$, viz. these are

$$
\begin{array}{ll}
\text { Order } & =3(n-1)(3 n-2), \\
\text { Class } & =6(n-1)^{2}, \\
\text { Nodes } & =\frac{3}{2}(n-1)\left(27 n^{3}-63 n^{2}+22 n+16\right), \\
\text { Cusps } & =3(n-1)(7 n-8), \\
\text { Double tangents } & =\frac{3}{2}(n-1)\left(12 n^{3}-36 n^{2}+19 n+16\right), \\
\text { Inflexions } & =12(n-1)(n-2),
\end{array}
$$

and as a verification it is to be observed, that the deficiency of the curve $\Delta$ is equal to that of the curve $J$, viz. it has the value $\frac{1}{2}(3 n-4)(3 n-5)$. The foregoing numbers include the result that the number of the binodal curves

is

$$
\sqrt{ }(f U)+\sqrt{ }(g V)+\sqrt{ }(h W)=0,
$$

$$
=\frac{3}{2}(n-1)\left(27 n^{3}-63 n^{2}+22 n+16\right) \text {. }
$$


The proof depended on the assumption, that to the points $(f, g, h)$ which lie on the curve $\Delta$, correspond the conics $\frac{f}{x}+\frac{g}{y}+\frac{h}{z}=0$ which touch the curve $\Sigma$; this M. Cremona proves in a very simple manner: the points of $J$ correspond each to each with the points of $\Sigma$, or if we please they correspond each to each with the tangents of $\Sigma$. To the $6 n(n-1)$ intersections of $J$ with any curve $\Omega$ (viz. $\sqrt{ }(f U)+\sqrt{ }(g V)+\sqrt{ }(h W)=0)$ correspond the $6 n(n-1)$ common tangents of $\Sigma$ and the conic $\frac{f}{x}+\frac{g}{y}+\frac{h}{z}=0$; if $\Omega$ has a node, two of the $6 n(n-1)$ intersections coincide, and the corresponding two tangents will also coincide, that is $\Omega$ having a node (or the point $(f, g, h)$ being on the curve $\Delta$ ), the conic touches the curve $\Sigma$. But it is not uninteresting to give an independent analytical proof. Write for shortness

$$
\begin{aligned}
& d U=A d x+B d y+C d z \\
& d V=A^{\prime} d x+B^{\prime} d y+C^{\prime} d z \\
& d W=A^{\prime \prime} d x+B^{\prime \prime} d y+C^{\prime \prime} d z
\end{aligned}
$$

and let $(x, y, z)$ be the coordinates of a point on $J,(X, Y, Z)$ those of the corresponding point on $\Sigma,(f, g, h)$ those of the corresponding point on $\Delta$. Write also for shortness

$$
B C^{\prime}-B^{\prime} C, C A^{\prime}-C^{\prime} A, A B^{\prime}-A^{\prime} B=P: Q: R,
$$

then we have

$$
\begin{aligned}
& A X+B Y+C Z=0, \\
& A^{\prime} X+B^{\prime} Y+C^{\prime} Z=0 \text {, } \\
& A^{\prime \prime} X+B^{\prime \prime} Y+C^{\prime \prime} Z=0 \text {, } \\
& { }^{A} \sqrt{ }\left(\frac{f}{U}\right)+B \sqrt{ }\left(\frac{g}{V}\right)+C \sqrt{ }\left(\frac{h}{W}\right)=0, \\
& A^{\prime}, \quad+B^{\prime}, \quad+C^{\prime}, \quad=0 \text {, } \\
& A^{\prime \prime},+B^{\prime \prime},+C^{\prime \prime},=0 \text {, }
\end{aligned}
$$

giving $A, B, C=0$, which is in fact the equation of the curve $J$; and moreover $A^{\prime}, \quad B^{\prime}, C^{\prime \prime}$ $A^{\prime \prime}, \quad B^{\prime \prime}, \quad C^{\prime \prime}$

giving $X: Y: Z=P: Q: R$, to determine the point $(X, Y, Z)$ on $\Sigma$; and

$$
\sqrt{\left(\frac{f}{U}\right)}: \sqrt{ }\left(\frac{g}{V}\right): \sqrt{ }\left(\frac{h}{W}\right)=P: Q: R,
$$

or, what is the same thing, $f: g: h=P^{2} U: Q^{2} V: R^{2} W$, to determine the point $(f, g, h)$ on $\Delta$. Treating now $(f, g, h)$ as constants, and $(X, Y, Z)$ as current coordinates, the conic $\frac{f}{X}+\frac{g}{Y}+\frac{h}{Z}=0$, will touch the curve $\Sigma$ at the point $(P, Q, R)$, if only the 
equation of the conic is satisfied by these values and by the consecutive values $P+d P, Q+d Q, R+d R$; or what is the same thing, if we have

$$
\begin{gathered}
\frac{f}{P}+\frac{g}{Q}+\frac{h}{R}=0 \\
\frac{f d P}{P^{2}}+\frac{g d Q}{Q^{2}}+\frac{h d R}{R^{2}}=0
\end{gathered}
$$

that is

$$
\frac{f}{P^{2}}: \frac{g}{Q^{2}}: \frac{h}{R^{2}}=Q d R-R d Q: R d P-P d R: P d Q-Q d P .
$$

If the functions on the right-hand side are as $U: V: W$, then these equations give

$$
f: g: h=P^{2} U: Q^{2} V: R^{2} W,
$$

that is $(f, g, h)$ will be a point on the curve $\Delta$. It is therefore only necessary to show that in virtue of the equation $J=0$ of the curve $J$, and of the derived equation $d J=0$, we have

$$
Q d R-R d Q: R d P-P d R: P d Q-Q d P=U: V: W .
$$

Take for instance the equation

$$
V(Q d R-R d Q)-U(R d P-P d R)=0,
$$

that is

$$
d R(U P+V Q+W R)-R(U d P+V d Q+W d R)=0,
$$

and this, and the other two equations will be satisfied if only $U P+V Q+W R=0$, $U d P+V d Q+W d R=0$; we have, neglecting a numerical factor,

$$
\begin{aligned}
& U=A x+A^{\prime} y+A^{\prime \prime} z, \\
& V=B x+B^{\prime} y+B^{\prime \prime} z, \\
& W=C x+C^{\prime} y+C^{\prime \prime} z,
\end{aligned}
$$

whence, attending to the values of $P, Q, R$, we have

$$
U P+V Q+W R=z J=0 ;
$$

hence also

$$
U d P+V d Q+W d R+(P d U+Q d V+R d W)=0
$$

so that

$$
U d P+V d Q+W d R=0
$$

if only

$$
P d U+Q d V+R d W=0
$$


and substituting for $P, Q, R, d U, d V, d W$ their values, the left-hand side is $=-J d z$, which is $=0$; hence the equations in question are proved, and $(f, g, h)$ is a point on the curve $\Delta$.

It is to be noticed, that the two curves $\Sigma, \Delta$ are geometrically connected through the three arbitrary points as follows: viz. taking as axes the sides of the triangle formed by these three points, then starting from any point $(f, g, h)$ of $\Delta$, we take the inverse point $\left(\frac{1}{f}, \frac{1}{g}, \frac{1}{h}\right)$, the harmonic line thereof $f x+g y+h z=0$, and finally the inverse conic $\frac{f}{x}+\frac{g}{y}+\frac{h}{z}=0$, which by what precedes touches $\Sigma$ in the point corresponding to the assumed point $(f, g, h)$ of $\Delta$ : and conversely starting with an assumed point on $\Sigma$, we take the conic $\frac{f}{x}+\frac{g}{y}+\frac{h}{z}=0$ which passes through the angles of the triangle and touches $\Sigma$ at the assumed point; the inverse line $f x+g y+h z=0$; the harmonic point $\left(\frac{1}{f}, \frac{1}{g}, \frac{1}{h}\right)$ of this line; and finally the inverse point $(f, g, h)$, which will be on the curve $\Delta$, the point corresponding to the assumed point on the curve $\Sigma$. 